\title{
Macroscopic Findings Severity
}

National Cancer Institute

\section{Source}

National Cancer Institute. Macroscopic Findings Severity. NCI Thesaurus. Code C119868.

The degree of something undesirable as determined by macroscopic findings. 